\title{
A MAXIMAL CHAIN APPROACH TO TOPOLOGY AND ORDER
}

\author{
R. VAINIO \\ Department of mathematics \\ Åbo Akademi \\ SF-20500 Åbo, Finland \\ (Received May 18, 1987 and in revised form August 26, 1987)
}

\begin{abstract}
On ordered sets (posets, lattices) we regard topologies (or, more general convergence structures) which on any maximal chain of the ordered set induce its own interval topology. This construction generalizes several well-known intrinsic structures, and still contains enough to produce interesting results on for instance compactness and connectedness. The "maximal chain compatibility" between topology (convergence structure) and order is preserved by formation of arbitrary products, at least in case the involved order structures are conditionally complete lattices.
\end{abstract}

AMS Subject Classification Codes: Primary 54A20, Secondary 54F05.

Key words and phrases: Intrinsic topologies (convergence structures) on ordered sets, order convergence, maximal chains.

\section{INTRODUCTION}

Parts of $\S \S 2$ and 3 of the present note can be regarded as an extension of Vainio [9]. Terminology is as in [9]; crucial definitions and notations are recapitulated in next section. Let $P$ be a partially ordered set (short: poset), endow $P$ with a topology (or, more generally: a convergence structure) $q$ such that all maximal chains of $P$ inherit their own interval topologies. The resulting space $(P, q)$ is called an $i$-space, and the structure $q$ an $i$-structure ( $i$-topology, or $i$-convergence) on $P$.

Specially on lattices, $i$-space compatibility provides an excellent realm for study of i.a. connectedness and compactness ( $§ 3)$. Partly, this is because completeness (conditional completeness) can be equivalently described as completeness (conditional completeness) of all maximal chains.

Examples of $i$-spaces abound. Whenever a poset is endowed with a topology, which is finer than interval topology and for which all maximal chains are compact sets, $i$ space compatibility follows. Every poset admits a finest $i$-convergence, as well as a finest $i$-topology. Several well-known intrinsic convergences are $i$-structures, as demonstrated in $\S 2$.

For conditionally complete lattices, an arbitrary product of $i$-spaces is again an $i$-space $(\S 4)$. This is of interest, because intrinsic topologies (convergences) of ordered structures do not behave well visavi formation of products (cf. Erné [1]). In $\S 4$ a few category theoretical remarks on $i$-convergences are included. 
The use of convergence structures instead of (the more special) topologies is motivated by works of i.a. M. Erné and D. C. Kent, which show a filter theoretical approach to intrinsic topologies on ordered sets provides an elegant and powerful method. Several important and natural structures, such as order convergence (cf. §1) are not topological. Moreover, R. N. Ball has created completion theory for lattices using Cauchy structures (i.e. Cauchy filters of uniform convergence structures) - still another example where classical topology does not suffice.

\section{PRELIMINARIES}

A convergence (structure) on a set $S$ is a map $q: S \rightarrow 2^{F(S)}(F(S) \equiv$ the set of all proper filters on $S$ ), which for all $x \in S$ satisfies

(1) $[x] \in q(x) . \quad([x]$ is the trivial ultrafilter derived from $x$.)

(2) $\mathcal{F} \in q(x)$ and $\mathcal{G} \supseteq \mathcal{F} \Rightarrow \mathcal{G} \in q(x)$.

(3) $\mathcal{F} \in q(x) \Rightarrow \mathcal{F} \cap[x] \in q(x)$.

Since we wish order convergence (see below) to constitute a special example of our theory, we can not assume $\mathcal{F}, \mathcal{G} \in q(x) \Rightarrow \mathcal{F} \cap \mathcal{G} \in q(x)$. The definition of convergence as stated in axioms (1), (2), (3) above dates back to Kent [5]. The couple $(S, q)$ is called a convergence space.

For $A \subseteq S$, let $q_{A}$ denote the convergence $q$ inherited to $A$. Of course, all topologies are convergences. The topological modification top $(q)$ is the finest topology on $S$ coarser than $q$. The category of convergence spaces (morphisms: continuous maps) is cartesian closed, a fact we will apply in $\S 4$. In Gähler [3] convergence spaces are treated in considerable depth. Following [3] and Vainio [9] a convergence space $(S, q)$ is called connected, if the topological modification $(S, \operatorname{top}(q))$ is, i.e. if all continuous maps from $(S, q)$ to the twopoint discrete space are constant maps. A set in a given convergence space is a connected set, if the corresponding subspace is a connected convergence space.

Partially ordered sets (posets) will be denoted by $P$, lattices by $L$. Quasi-ordered sets (no anti-symmetry assumed!) will be used in $§ 4$, primarily as tools. Totally ordered subsets of $P$ are called chains; a chain which is not a proper subset of any other chain of $P$ is called a maximal chain in $P$. For $A \subseteq P, A^{\star}\left(A^{+}\right)$is the set of all upper bounds (lower bounds) of $A$. If $A=\{a\}$, we write $a^{\star}\left(a^{+}\right)$. A poset is order dense, if $] x, y[\neq \emptyset$ for all $x<y$. Definitions of complete (conditionally complete) lattices and subcomplete (conditionally subcomplete) sublattices are the standard ones. The lattice translations $L \rightarrow L$ are the maps $x \rightarrow a \vee x, x \rightarrow a \wedge x$, for any $a \in L$. Any chain $J$ in $L$ is, of course, a lattice in its own right, and for $S \subseteq J$ the indices in the expressions $\vee_{J} S, \vee_{L} S$ tell in which lattice the l.u.b. is formed, provided it exists (dual notation for g.l.b.).

A poset endowed with a convergence is called $T_{1}$-ordered, if all sets $a^{\star}, a^{+}$are closed. We will use several intrinsic convergence structures on a given poset $P$, i.a. interval topology $t(P)$ (O. Frink) and order convergence $o(P)$ (D. C. Kent). The former is defined as the coarsest $T_{1}$-ordered topology on $P$, and the latter is given by

$\mathcal{F} \in o(P)(x) \Leftrightarrow \wedge \mathcal{F}^{\star}$ and $\vee \mathcal{F}^{+}$both exist, and both equal $x$. 
Hereby,

$$
\mathcal{F}^{\star}=\cup\left\{F^{\star}: F \in \mathcal{F}\right\} \text { and } \mathcal{F}^{+}=\cup\left\{F^{+}: F \in \mathcal{F}\right\}
$$

In any lattice $L$, the classical order topology (G. Birkhoff) equals top $(o(L))$.

We are now ready to proceed to ordered topological spaces and their maximal chains.

\section{DEFINITION AND EXAMPLES OF $i$-SPACES}

Let $q$ be a convergence on an arbitrary poset $P$. The pair $(P, q)$ is an $i$-space $(q$ is an $i$-convergence, or an $i$-topology on $P$ ), if for any maximal chain $J$ of $P$ the equality $t(J)=q_{J}$ holds. Since interval topologies on chains are Hausdorff (even $\left.T_{5}\right)$, any $i$-space (P.q) satisfies

$x \leq y$ and $\mathcal{F} \in q(x) \cap q(y) \Rightarrow \mathcal{F}$ has no trace to any chain containing $x$ and $y$. We also note any $T_{1}$-ordered space $(P, q)$ is an $i$-space, if and only if $t(J) \geq q_{J}$ for all maximal chains $J$ of $P$. However, there are $i$-spaces which are not $T_{1}$-ordered. Clearly: on any poset all $T_{1}$-ordered topologies yielding compact maximal chains are automatically $i$-topologies.

EXAMPLE 1. Every poset $P$ admits a finest $i$-convergence $s(P)$, which determines Rennie's chain topology $r(P)$. (A set $S$ in $P$ is $r(P)$-open, if and only if $S \cap J$ is a $t(J)$-open set for all maximal chains $J$. This structure was referred to by Rennie [7].) Of course, $r(P)$ is the finest $i$-topology on $P$. In general, there is no coarsest $i$-convergence or coarsest $i$-topology on a given poset. Indeed, let $\mathcal{S}_{1}\left(\mathcal{S}_{2}\right)$ be the set of all open angular regions in the plane $\mathbf{R}^{2}$ with vertex at $(1,0)($ at $(0,1))$. The restriction of $\mathcal{S}_{1}\left(\mathcal{S}_{2}\right)$ to $] 0,1[\times] 0,1[$ determines the topology $\tau_{1}\left(\tau_{2}\right)$ on $] 0,1[\times] 0,1\left[\right.$. Now, both $\tau_{1}$ and $\tau_{2}$ are $i$-topologies on the poset $] 0,1[\times] 0,1[$ (endowed with natural order), but neither their g.l.b. topology nor their g.l.b. convergence are $i$-structures.

EXAMPLE 2. Among all $i$-topologies on a given poset $P$ admitting closed maximal chains, there is a coarsest one denoted by $w(P)$. Indeed, given the maximal chains $J$ of $P$, let $w(P)$ arise from the sub-base of closed sets $\left\{a^{\star} \cap J, a^{+} \cap J: a \in J\right\}$. It is easily seen $w(P)$ has the desired properties; also note that for $P \equiv] 0,1[\times] 0,1\left[\right.$, no set of the form $a^{\star}$ or $a^{+}$is $w(P)$-closed. There are even $i$-spaces in which no maximal chain is closed, cf. the lattice $\{0, a, b, 1\}, a$ and $b$ non-related, endowed with the topology whose closed sets are generated by $\{a, b\},\{0\},\{1\}$. We note that if the maximal chains of an $i$-space $(P, q)$ are closed sets, then top $(q)$ is an $i$-topology on $P$.

The following example shows that on posets, which are not lattices, order convergence misbehaves.

EXAMPLE 3. Let $P$ be the subposet of $\mathbf{R}^{2}$ consisting of the strictly negative part of the $x$-axis, all $y$-axis for $y \geq 1$, and the point $(1,0)=\alpha$. Clearly, $o(P)(\alpha)=\{[\alpha]\}$, which means the restriction of $o(P)$ to the maximal chain containing $\alpha$ is strictly finer than the interval topology of that chain.

From now on, in this section we will restrict our attention to lattices only. Our first aim is to generalize two lemmata of Vainio [9], originally proved assuming conditional 
completeness. First we mention the obvious

LEMMA 4. Let $L$ be any lattice and $\mathcal{F}$ a filter on $L$ arising from some filter base $\mathcal{B}$ of sets $B$ for which $\vee_{L} B$ and $\wedge_{L} B$ exist. Then, for all $x \in X$

$\mathcal{F} \in o(L)(x) \Leftrightarrow \vee_{L}\left\{\wedge_{L} B: B \in \mathcal{B}\right\}, \wedge_{L}\left\{\vee_{L} B: B \in \mathcal{B}\right\}$ both exist and both

equal $x$.

LEMMA 5 (cf. Vainio [9, Lemma 3]). Let $L$ be any lattice, $J$ a maximal chain of $L$, and $S$ a subchain of $J$. Then,

$\vee_{J} S$ exists $\Rightarrow \vee_{L} S$ exists and equals $\vee_{J} S$, and dually.

PROOF. Assume $\vee_{J} S=\alpha, \alpha \notin S$, and let $\beta \leq \alpha$ be an $L$ upper bound of $S$. Then, $s \leq \beta \leq \alpha$ for all $s \in S$. Of course, $\beta \notin S$. Thus, $\beta<\alpha$ would mean $J$ is not a maximal chain. Hence $\beta=\alpha$, and we have revealed $\alpha$ is the smallest $L$ upper bound of $S . \diamond$

Example 3 shows Lemma 5 cannot be extended to posets.

LEMMA 6. (cf. Vainio [9, Lemma 7]). For an arbitrary lattice L, any convergence structure between $t(L)$ and $o(L)$ is an $i$-structure.

PROOF. Let $J$ be any maximal chain of $L$, take $x \in J$, and denote the $t(J)$ neighborhood filter of $x$ by $\mathcal{F}$. Since $t(J)$ equals $o(J)$, Lemma 4 gives $\mathcal{F}$ has a base $\mathcal{B}$ consisting of bounded $J$-intervals $B$ such that $\vee_{J}\left\{\wedge_{J} B: B \in \mathcal{B}\right\}$ and $\wedge_{J}\left\{\vee_{J} B: B \in \mathcal{B}\right\}$ both exist and both equal $x$. (Note that any bounded $J$-interval possesses l.u.b. and g.l.b. in $J$.) According to Lemma 5 , the index $L$ can replace the index $J$ in the above expressions, and thus, $\mathcal{F}$ is a base of a filter on $L$ order-converging towards $x$. Hence, $o(L)$ is an $i$-structure. Since $t(L)_{J} \geq t(J)$ and $t(L) \leq o(L)$, we get $t(L)$ is an $i$-structure, too.॰

COROLLARY 7. On every lattice $L, o(L) \leq s(L)$. This result is not true for arbitrary posets (cf. Ex. 3).

EXAMPLE 8. In an arbitrary lattice, there is a host of well-known convergence structures in between interval topology and order convergence. Erné [2] mentions among the topologies, e.g. interval topology, new interval topology, Lawson topology, bi-Scott topology, Rennie's L-topology, lim-inf topology, Birkhoff's order topology, and among the convergences (through which some of the previous topologies can be described), e.g. interval convergence, lim-inf convergence, and order convergence. In view of Lemma 6 , we know all of them are $i$-structures.

The remainder of the paper will give further motivation for investigating $i$-spaces.

\section{COMPACTNESS AND CONNECTIVITY}

Let $L$ be an arbitrary lattice. We say a property $p$ of $L$ is described by maximal chains, if

$L$ satisfies $p \Leftrightarrow$ All maximal chains of $L$ satisfy $p$.

Kogalovskiu [6] proves completeness is described by maximal chains. We will note Lemma 5 and Rennie [7, Theorem 1] together imply that conditional completeness is described by maximal chains (also, cf. Vainio [9]), and from this Kogalovskiı̌'s result follows. 
LEMMA 9. In any lattice, the following properties are described by maximal chains: (1) convexity, (2) order density, (3) conditional completeness, (4) completeness.

PROOF. (3) Assume all maximal chains of $L$ are conditionally complete, let $S$ be a chain of $L$ with upper bound $m$, and let $J$ be a maximal chain containing $S$ and $m$. Hence $\vee_{L} S$ exists (Lemma 5 ), and $\mathrm{L}$ is conditionally complete according to Rennie [7, Theorem 1]. The converse statement is trivial (cf. for instance Vainio [9, Lemma 3]). (4) Assume all maximal chains of $L$ are complete, and let $J_{1}$ and $J_{2}$ be maximal chains with greatest elements $a_{1}, a_{2}$. Since $a_{1} \vee a_{2} \in J_{1} \cap J_{2}$, then all maximal chains have the same greatest element, which proves $L$ has a greatest element. Applying (3) above the proof is completed. $\diamond$

COROLLARY 10. A lattice is (conditionally) complete, if and only if all maximal chains of the lattice are (conditionally) subcomplete.

Using i.a. Lemma 9 and the well-known fact that any chain $J$ is complete (conditionally complete), if and only if $t(J)$ is compact (all bounded ultrafilters on $J$ are $t(J)$-convergent), we obtain the following two theorems.

THEOREM 11. For $q$ an $i$-convergence on any lattice $L$, the following are equivalent:

(1) $L$ is complete.

(2) $(L, q)$ has compact maximal chains.

If $q \leq t(L)$, but all maximal chains of $(L, q)$ still are closed sets, then expressions (1) and (2) are equivalent to

(3) $(L, q)$ is compact.

PROOF. For part (3), use the well-known fact that a lattice is complete, if and only if it is compact in its interval topology. $\diamond$

For $L$ an arbitrary lattice, denote by $w(L)$ the coarsest $i$-convergence on $L$ admitting closed maximal chains ( $w(L)$ is always a topology, cf. Ex. 2), and take $w(L) \leq q \leq s(L)$.

THEOREM 12. A lattice $L$ is (conditionally) complete, if and only if all (bounded) ultrafilters on $L$ containing some maximal chain of $L$ are $q$-convergent.

We next improve Theorem 4 of Vainio [9]. Proofs are omitted; the reader is referred to the well-known fact that any chain $J$ is conditionally complete and order dense, if and only if $t(J)$ is connected, to Lemma 9, and to the proof of [9, Theorem 4]. Note that any convex subspace of an $i$-space is again an $i$-space. Definitions regarding connectivity of convergence spaces are as in Gähler [3] or Vainio [9]. Below, $L$ is always a lattice.

THEOREM 13. Every conditionally complete, order dense, convex sublattice of an $i$-space $(L, q)$ is connected.

THEOREM 14. Let $L$ be conditionally complete, and let $(L, q)$ be a $T_{1} i$-space in which all translations are continuous maps. Then, all connected components of $(L, q)$ are order dense, convex (and hence, conditionally complete) sublattices of $L$.

COROLLARY 15. In conditionally complete $T_{1} i$-spaces $(L, q)$ with continuous translations, the connected components can be described as the maximal order dense, convex 
sublattices of $L$.

Let $X \equiv(X, q)$ be a convergence space and recall that $(X, q)$ and $(X, \operatorname{top}(q))$ yield the same real-valued continuous maps, denoted by $C(X)$. It is known (cf. Stone [8] ) that the lattice $C(X)$ is conditionally complete, if and only if $\operatorname{top}(q)$ is extremally disconnected. We next characterize conditional completeness of $C(X)$ in terms of any $i$-structure $q$ on $C^{\prime}(X)$.

THEOREM 16. The lattice $C(X)$ is conditionally complete, if and only if all maximal chains in $C(X)$ are $q$-connected sets.

The proof is an easy consequence of Lemma 9 and of the fact that $C(X)$ is order dense.

\section{PRODUCTS OF $i$-SPACES}

Category theoretical definitions are as in Herrlich [4].

At first, we will regard a somewhat more general (but from a category theoretical point of view more natural) situation than $i$-space compatibility. The results will then be applied to i.a. formation of products of $i$-spaces.

Let $S$ be a quasi-ordered set endowed with a convergence $q$ such that for any maximal chain $J$ of $S$ the inequality $q_{J} \geq t(J)$ holds true. The corresponding category (morphisms: increasing continuous maps) is denoted by $Q C O N$ and the full subcategory determined by the partially ordered objects is called PCON. The first category is mainly a tool, used to obtain Remark 18 below.

LEMMA 17. The concrete category QCON is topological.

PROOF. Let $\left(S_{i}, q_{i}\right)_{i \in I}$ be an arbitrary family of $Q C O N$-objects, consider a set $S$ and set theoretical maps $f_{i}: S \rightarrow S_{i}, i \in I$. Then, for any $x, y \in S$, define

$x \leq y$ in $S \Leftrightarrow f_{i}(x) \leq f_{i}(y)$ in $S_{i}$, all $i \in I$.

$\mathcal{F} \in q(x) \Leftrightarrow f_{i}(\mathcal{F}) \in q_{i}\left(f_{i}(x)\right)$, all $i \in I$.

Obviously, $S$ is thus endowed with a quasi-order $\leq$ and a convergence $q$. It remains to prove the resulting space is a $Q C O N$-object. Therefore, let $J$ be a maximal chain in $S$, and let $J_{i}$ be one maximal chain in $S_{i}$ containing the chain $f_{i}(J)$. For any $i \in I$, denote the restriction of $f_{i}$ to $J$ by $g_{i}$, and note that for $\alpha \in J$

$$
\alpha^{+}=\cap_{i \in I} g_{i}^{-1}\left(g_{i}(\alpha)^{+}\right)
$$

where $\alpha^{+}$and $g_{i}(\alpha)^{+}$are formed in the chains $J$ and $J_{i}$, respectively. Since all maps $g_{i}$ are continuous, the sets $\alpha^{+}$(and dually, $\alpha^{\star}$ ) are closed sets in $q_{J}$, and hence $q_{J} \geq t(J)$. $\diamond$

There are interesting consequences of Lemma 17. Since $Q C O N$ is a topological category, Herrlich [4; Section 2.1, T(5)] quite easily gives $Q C O N$ is a cartesian closed category. Power objects in $Q C O N$ are the spaces $C I_{c}(S, T)$, i.e. the set of $Q C O N$-morphisms (between given $Q C O N$-objects $S$ and $T$ ) endowed with product order and continuous convergence structure. Indeed, we obtain (all proofs omitted)

REMARK 18. The topological category $Q C O N$ is cartesian closed, and contains PCON as a quotient-reflective subcategory; hence $P C O N$ is cartesian closed. 
Needless to say, Lemma 17 and Remark 18 do not apply to $i$-space compatibility.

It is an immediate consequence of the proof of Lemma 17 that QCON (and thus, $P C O N$ ) is closed under formation of arbitrary products and subspaces. This implies a few remarks on orderability. We call a convergence space $p$-orderable, if there is a partial order on $X$ which makes $(X, q)$ a $P C O N$-object. (If the po-structure in question is a total order and $q$ a topology, we get the well-known orderable spaces.) Then let PORD denote the full subcategory of the convergence space category $C O N$ consisting of all p-orderable spaces. Since $C O N$ is a topological category, any subcategory of this category which is closed under formation of products and subspaces is reflective in it. Thus, PORD is a reflective subcategory of $C O N$.

The remainder of the paper deals with a special construction; products of $i$-spaces. Consider a family $\left(P_{i}, q_{i}\right)_{i \in I}$ of $i$-spaces, each $P_{i}$ being a poset and each $q_{i}$ a convergence. Denote the product space by $(P, q)$, i.e. $P$ is the product poset and $q$ the product convergence. Since $P C O N$ is closed under formation of arbitrary products, $(P, q)$ is an $i$-space, if and only if $\Pi q_{i} \leq s(P)$. This condition is equivalent to continuity of all projections $p r_{\imath}:(J, t(J)) \rightarrow\left(P_{i}, q_{i}\right)$, where $J$ is an arbitrary maximal chain of $P$ and $i \in I$. This is satisfied at least, if for all $i, J$ as above, each set $p r_{i}(J)$ is a convex chain in $P_{i}$ (i. e. $p r_{\imath}(J)$ is a convex subset of any maximal chain of $P_{i}$ containing it). The following lemma gives a natural sufficient condition.

Let $\left(L_{i}\right)_{i \in I}$ be conditionally complete lattices, denote $L \equiv \Pi L_{i}$, and let $J$ be a maximal chain of $L$.

LEMMA 19. For each $i \in I, p r_{i}(J)$ is a convex chain in $L_{i}$.

PROOF. Assume $p r_{\alpha}(J)$ not convex, and let $h_{\alpha}$ be a "hole" in it. Define the subset $A$ of $J$ by

$x \in A \Leftrightarrow x \in J$ and $p r_{\alpha}(x)>h_{\alpha}$.

Let $z \in L$ be the element defined by $p r_{i}(z)=\wedge p r_{i}(A)$ for $i \in I, i \neq \alpha$, and $p r_{\alpha}(z)=h_{\alpha}$. (The conditional completeness assumption implies $\wedge p r_{i}(A)$ exists.) We now prove $z \in J$ by showing $z$ is order related to an arbitrary $s \in J$.

If $p r_{\alpha}(s)>h_{\alpha}$, then $s \in A$, and for $i \neq \alpha p r_{i}(s) \geq \wedge p r_{i}(A)=p r_{i}(z)$, which gives $s>z$.

If $p r_{\alpha}(s)<h_{\alpha}$, then $s<x$ for all $x \in A$, and hence for $i \neq \alpha p r_{i}(s) \leq \wedge p r_{\imath}(A)=p r_{\imath}(z)$, and $s<z$ follows.

Now, $h_{\alpha}=p r_{\alpha}(z) \in p r_{\alpha}(J)$, a contradiction. $\diamond$

THEOREM 20. The product of given $i$-spaces $\left(L_{i}, q_{i}\right)_{i \in I}$ is an $i$-space, if every $L_{\imath}$ is a conditionally complete lattice. 


\section{REFERENCES}

1. M. ERNÉ, Topologies on products of partially ordered sets I-III, Algebra Univ. 11 (1980) no 3 pp. 295-311; ibid. 11 (1980) no 3, pp. 312-319; ibid. 13 (1981) no 1, pp. 1-23.

2. - and H. GATZKE, Convergence and continuity in partially ordered sets and semilattices, in Continuous Lattices and their Appl., Proc. Bremen 1982, M.Dekker.

3. W. GÄHLER, Grundstrukturen der Analysis I-II, Akademie-Verlag und Birkhäuser Verlag, 1977-78.

4. H. HERRLICH, Categorical topology 1971-1981, Proc. Symp. Gen. Top. Appl. Prague 1981, pp. 279-383.

5. D. C. KENT, On convergence groups and convergence uniformities, Fund. Math. 60 (1967), pp. 213-222.

6. S. R. KOGALOVSKIn, On linearly complete ordered sets (Russian), Uspehi Mat. Nauk. 19 (1964) no 2, pp. 147-150.

7. B. C. RENNIE, Lattices, Proc. London Math. Soc. 52 (1951), pp. 386-400.

8. M. H. STONE, Boundedness properties in function-lattices, Canad. J. Math. 1 (1949), pp. 176-186.

9. R. VAINIO, Connectedness properties of lattices, Canad. Math. Bull. 29 (1986) no 3, pp. 314320 . 


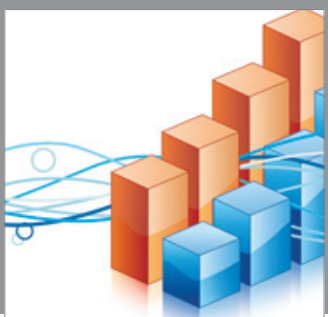

Advances in

Operations Research

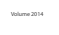

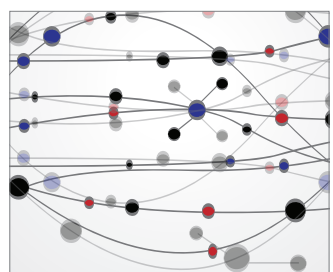

\section{The Scientific} World Journal
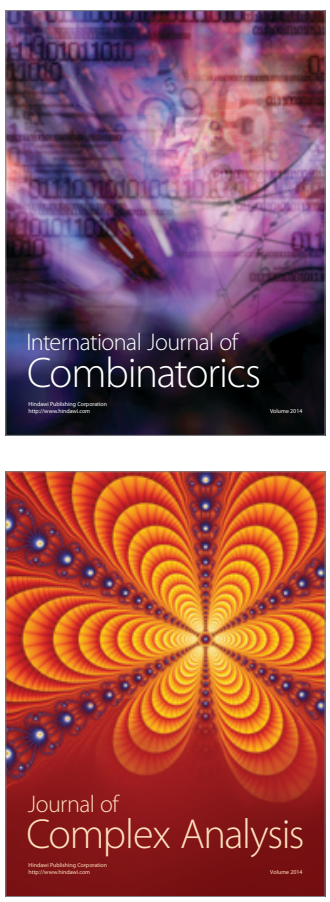

International Journal of

Mathematics and

Mathematical

Sciences
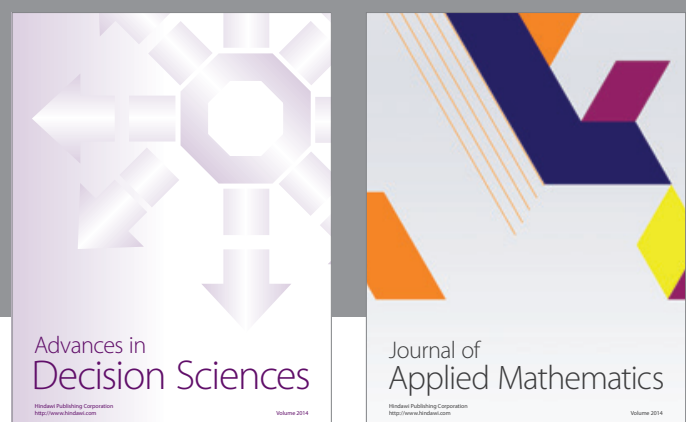

Journal of

Applied Mathematics
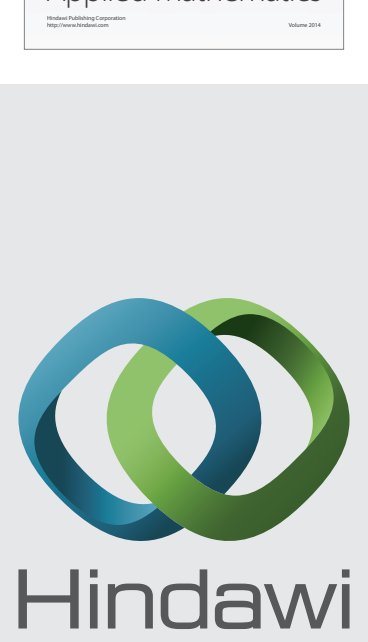

Submit your manuscripts at http://www.hindawi.com
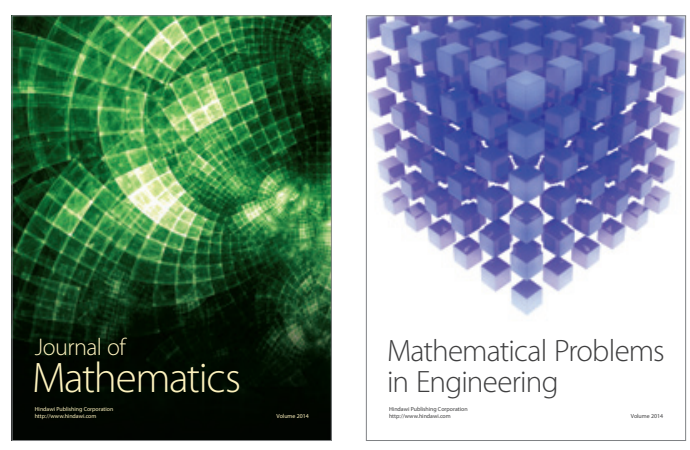

Mathematical Problems in Engineering
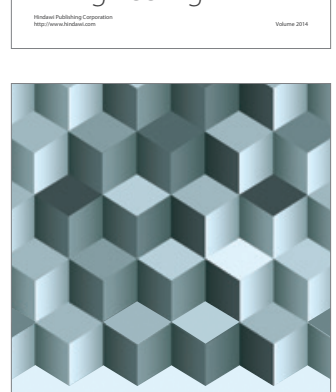

Journal of

Function Spaces
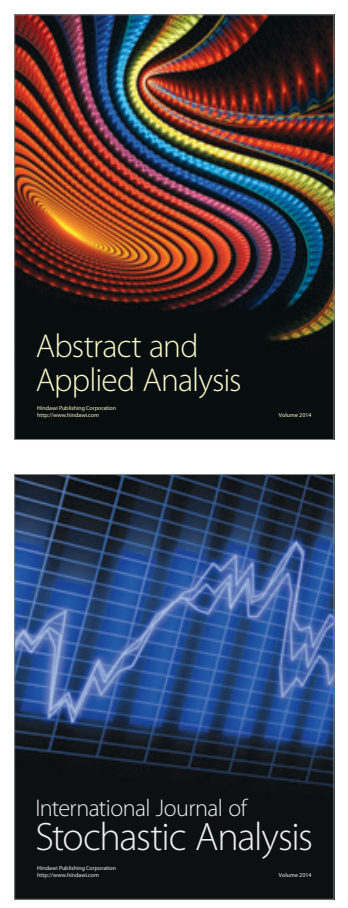

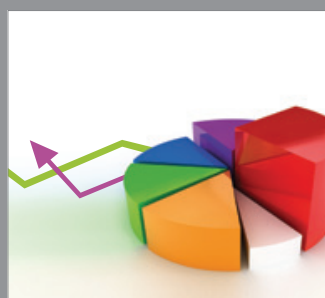

ournal of

Probability and Statistics

Promensencen
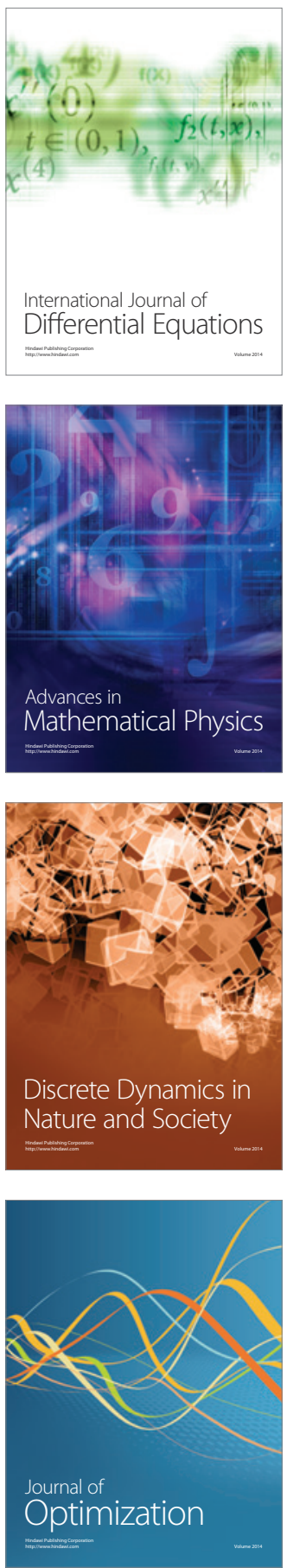